\title{
Fuzzy Ontology Model for Knowledge Management
}

\author{
Jun Zhai Lixin Shen Zhou Zhou Yan Liang \\ School of Economics and Management, Dalian Maritime University, Dalian 116026, P. R. China
}

\begin{abstract}
Ontology is the basis of sharing and reusing knowledge on the semantic web. The fuzzy ontology is an extension of the domain ontology for solving the uncertainty problems. Current fuzzy ontology models do not focus on essential semantic relationships between fuzzy concepts, which lead difficulty in ontology integrating. To represent formally the fuzzy knowledge, this paper proposes a series of fuzzy ontology models that consist of fuzzy domain ontology and fuzzy linguistic variable ontologies, considering semantic relationships of concepts, including set relation, order relation and equivalence relation. Application of the fuzzy ontology to transportation knowledge modeling shows that this research facilitates the knowledge share and reuse for fuzzy systems on the semantic web.
\end{abstract}

Keywords: Ontology, Fuzzy ontology, Fuzzy system, Knowledge management

\section{Introduction}

Ontology is a conceptualization of a domain into a human understandable, machine-readable format consisting of entities, attributes, relationships, and axioms [1]. It is used as a standard knowledge representation for the Semantic Web [2]. However, the conceptual formalism supported by typical ontology may not be sufficient to represent uncertainty information commonly found in many application domains due to the lack of clear-cut boundaries between concepts of the domains. Moreover, fuzzy knowledge plays an important role in many domains that face a huge amount of imprecise and vague knowledge and information, such as text mining, multimedia information system, medical informatics, machine learning, and human natural language processing [3].

To handle uncertainty of information and knowledge, one possible solution is to incorporate fuzzy theory into ontology. Then we can generate fuzzy ontologies, which contain fuzzy concepts and fuzzy memberships. The fuzzy ontologies are capable of dealing with fuzzy knowledge [4], and are efficient in text and multimedia object representation and retrieval [5].
Lee et al. [6] proposed an algorithm to create fuzzy ontology and applied it to news summarization. This work is based on their previous work on ontology-based fuzzy event extraction agents for Chinese news summarization [7]. Tho et al. proposed a Fuzzy Ontology Generation Framework (FOGA) for fuzzy ontology generation on uncertainty information [8]. This framework is based on the idea of fuzzy theory and Formal Concept Analysis (FCA). Abulaishet et al. [9] proposed a fuzzy ontology framework in which a concept descriptor is represented as a fuzzy relation which encodes the degree of a property value using a fuzzy membership function. To enable representation and reasoning for fuzzy ontologies, Kang et al. [10] proposed a new fuzzy extension of description logics called the fuzzy description logics with comparison expressions (FCDLs).

But, current fuzzy ontology models do not focus on essential semantic relationships between fuzzy concepts, which lead difficulty in ontology mapping and integrating. To represent formally the fuzzy knowledge, this paper proposes a new kind of fuzzy ontology models. The rest of this paper is organized as follows: Section 2 introduces fuzzy domain ontology model. Section 3 proposes fuzzy linguistic variable ontology model, extended fuzzy ontology model and basic fuzzy ontology model. Section 4 applies the fuzzy ontology to transportation knowledge modeling. Finally, section 5 concludes the paper.

\section{Fuzzy domain ontology model}

An ontology ( $O$ ) organizes domain knowledge in terms of concepts $(C)$, properties $(P)$, relations $(R)$ and axioms ( $A$ ), and can be formally defined as follows.

Definition 1 (Ontology) - An ontology is a 4tuple $O=(C, P, R, A)$, where:

1. $C$ is a set of concepts defined for the domain. A concept is often considered as a class in an ontology. 2. $P$ is a set of concept properties. A property $p \in P$ is defined as an instance of a ternary relation of the form $p(c, v, f)$, where $c \in C$ is an ontology concept, $v$ is a property value associated with $C$ and $f$ defines restriction facets on $v$. Some of the 
restriction facets are - type $\left(f_{t}\right)$, cardinality $\left(f_{c}\right)$, and range $\left(f_{r}\right)$. The type facet $f_{t}$ may be any one from the standard data types supported by ontology editors, i.e. $f_{t} \in$ \{boolean, integer, float, string, symbol, instance, class, $\ldots\}$. The cardinality facet $f_{c}$ defines the upper and lower limits on the number of values for the property. The range facet $f_{r}$ specifies a range of values that can be assigned to the property.

3. $R=\left\{r \mid r \subseteq C \times C \times R_{t}\right\}$ is a set of binary semantic relations defined between concepts in $C$. $R_{t}=$ \{one-to-one, one-to-many, many-to-many $\}$ is the set of relation type.

A set of basic relations is defined as \{synonym of, kind of, part of, instance of, property of $\} \subset R$ which have the following interpretations:

(1) $C_{i}$ synonym of $c_{j}: c_{i}$ is equivalent to $c_{j}$. The synonym relation of natural language is modeled in an ontology using the equivalence relation. If two concepts $C_{i}$ and $C_{j}$ are declared equivalent in an ontology then instances of concept $C_{i}$ can also be inferred as instances of $c_{j}$ and vice-versa.

(2) $C_{j}$ kind of $C_{i}: C_{i}$ is a generalization of $c_{j}$. When an ontology specifies that $C_{i}$ is a generalization of $c_{j}$, then $c_{j}$ inherits all property descriptors associated with $C_{i}$, and these need not be repeated for $C_{j}$ while specifying the ontology.

(3) $C_{j}$ part of $C_{i}: C_{i}$ has part $C_{j}$. In an ontology, a concept which is defined as aggregation of other concepts is expressed using this relation.

(4) $C_{j}$ instance of $C_{i}: C_{j}$ is an instance of $C_{i}$.

(5) $C_{j}$ property of $C_{i}: C_{j}$ is a property of $C_{i}$.

4. $A$ is a set of axioms. An axiom is a real fact or reasoning rule.

The fuzzy ontology is created as an extension to the standard ontology.

Definition 2 (Fuzzy domain ontology) - A fuzzy domain ontology is a 4-tuple $O_{F}=\left(C, P_{F}, R_{F}, A_{F}\right)$, where:

1. $C$ is a set of concepts. Differing from definition 1, every concept here has some properties whose value is fuzzy concept or fuzzy set.

2. $P_{F}$ is a set of properties. A property $p_{F} \in P_{F}$ is defined as a 5-tuple of the form $p_{F}\left(c, v_{F}, q_{F}, f, U\right)$, where $c \in C$ is an ontology concept, $v_{F}$ represents property values,$q_{F}$ models linguistic qualifiers, which can control or alter the strength of a property value $v_{F}, f$ is the restriction facets on $v_{F}$, and $U$ is the universe of discourse. Both $v_{F}$ and $q_{F}$ are the fuzzy concepts at $U$, but $q_{F}$ changes the fuzzy degree of $v_{F}$. For example, "price" is a property of concept "fruit". The value of "price" may be either fuzzy concept "cheap" or fuzzy number "around 50", and the linguistic qualifiers may be "very", "little", "close to" etc. Therefore, the final value of "price" may be "very cheap" or "little expensive".

3. $R_{F}$ is a set of inter-concept relations between concepts. Like fuzzy concept properties, $r_{F} \in R_{F}$ is defined as a 5-tuple of the form $r_{F}\left(c_{1}, c_{2}, t, s_{F}, U\right)$, where $c_{1}, c_{2} \in C$ are ontology concepts, $t$ represents relation type, $U$ is the universe of discourse, and $S_{F}$ models relation strengths and is fuzzy concept at $U$, which can represent the strength of association between concept-pairs $\left\langle c_{1}, c_{2}\right\rangle$.

4. $A_{F}$ is a set of fuzzy rules. In a fuzzy system the set of fuzzy rules is used as knowledge base.

The fuzzy domain ontology is used to model domain expert knowledge. But, due to the lack of relationships between fuzzy concepts that can be the value of properties, it is difficult to integrate diverse ontology systems. For example, in an ontology the set of property "price" value is \{cheap, appropriate, expensive, ...\}, and in other ontology the same set is \{high, low, middle, ... \}. To map these ontologies, it is necessary to define the semantic relationship between fuzzy concepts, e.g. "cheap" and "expensive" have the relation of disjointness, and "low" and "high" have the same relation of disjointness etc.

Consequently, we propose the fuzzy linguistic variables ontology models.

\section{Fuzzy linguistic variable ontology}

The fuzzy linguistic variables proposed by Zadeh are the basic of fuzzy knowledge and fuzzy system [3]. To achieve the knowledge share and reuse for fuzzy systems on the semantic web, it is necessary to represent the fuzzy linguistic variables with ontology.

Definition 3 (Fuzzy linguistic variable) - Fuzzy linguistic variable is the variable whose value is term or concept in natural language. A fuzzy linguistic variable is a 4-tuple $(X, T, M, U)$, where:

1. $X$ is the name of fuzzy linguistic variable, e.g. "price" or "speed" etc. 
2. $T$ is the set of terms which is the value of fuzzy linguistic variable, e.g. $T=\{$ cheap, appropriate, expensive, ... $\}$ or $T=\{$ fast, middle, slow,... .

3. $M$ is the mapping rules which map every term of $T$ to fuzzy set at $U$.

4. $U$ is the universe of discourse.

Introducing semantic relationships between concepts, we obtain the ontology model.

Definition 4 (Fuzzy linguistic variable ontology) - A fuzzy linguistic variable ontology is a 5-tuple $O_{F}=\left(c_{a}, C_{F}, R, F, U\right)$, where:

1. $C_{a}$ is a concept on the abstract level, e.g. “price”, "speed” etc. The corresponding element of $c_{a}$ is $X$ in definition 3 .

2. $C_{F}$ is the set of fuzzy concepts which describes all values of $c_{a}$. The corresponding element of $C_{F}$ is $T$ in definition 3 , but $C_{F}$ has certain structure or relations $R$.

3. $R=\left\{r \mid r \subseteq C_{F} \times C_{F}\right\}$ is a set of binary relations between concepts in $C_{F}$. A kind of relation is set relation $R_{S}=$ inclusion, intersection, disjointness, complement $\}$, and the other relations are the order relation and equivalence relation $R_{O}=\{\leq, \geq,=\} . C_{F}$ and an order relation $r$ compose the ordered structure $<C_{F}, r>$.

4. $F$ is the set of membership functions at $U$, which is isomorphic to $C_{F}$. The corresponding element of $F$ is $M$ in definition 3 , but $F$ has also certain structure or relations.

5. $U$ is the universe of discourse.

Definition 4 is more complex than definition 3 in order to describe the semantic information.

Modeling the linguistic qualifiers, we extend the fuzzy linguistic variable ontology as follows.

Definition 5(Extended fuzzy ontology) -An extended fuzzy ontology is a 7-tuple $O_{F}=\left(c_{a}, C_{F}, R, F, Q, O, U\right)$, where:

1. $C_{a}, C_{F}, R, F, U$ have same interpretations as defined in definition 4.

2. $Q$ is the set of the linguistic qualifiers, e.g. $Q=\{$ very, little, close to, ... $\}$. An qualifier $q \in Q$ and a fuzzy concept $c_{F} \in C_{F}$ compose a composition fuzzy concept that can be the value of $c_{a}$, e.g. "very cheap".

3. $O$ is the set of fuzzy operators at $U$, which is isomorphic to $Q$.
To simplify the transform from fuzzy linguistic variables to fuzzy ontology, we introduce the basic fuzzy ontology model as follows.

Definition 6 (Basic fuzzy ontology) -A basic fuzzy ontology is a 4-tuple $O_{F}=\left(c_{a}, C_{F}, F, U\right)$, where $C_{a}, C_{F}, F, U$ have same interpretations as defined in definition 4 , which satisfy the following conditions:

1. $C_{F}=\left\{c_{1}, c_{2}, \cdots, c_{n}\right\}$ is a limited set.

2. Only one relation of set, the relation of disjointness, exists in $C_{F}$, and $C_{F}$ is complete at $U$. In the other words, $C_{F}$ is a fuzzy partition of $U$.

3. $C_{F}$ has an ordered relation $\leq$, and $\left\langle C_{F}\right.$, $\leq>$ is a complete ordered set, i.e. all concepts in $C_{F}$ constitute a chain $c_{1} \leq c_{2} \leq \cdots \leq c_{n}$

4. $F$ is optional element of ontology.

An example of basic fuzzy ontology is $O_{F}=$ ( $c_{a}=$ price of fruit, $C_{F}=$ \{very cheap, cheap, appropriate, expensive, very expensive , $U=[0,100]$ ) .

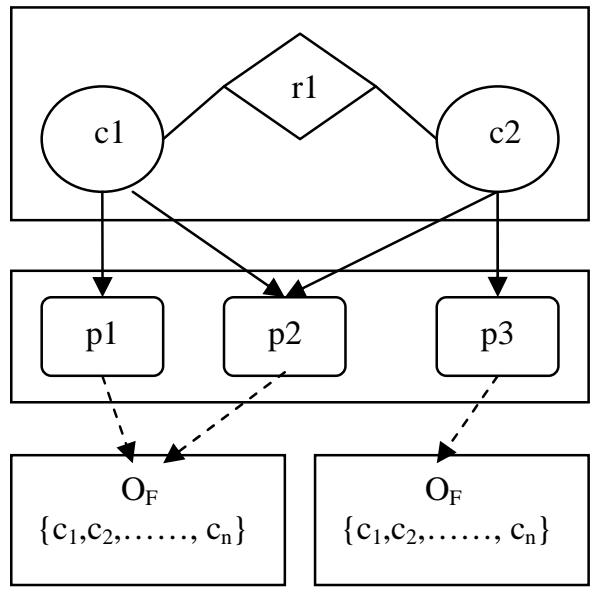

c: concept r: relation $\quad$ p: property $\mathrm{O}_{\mathrm{F}}$ : fuzzy ontology property of value of

Fig. 1: Three-layered ontology structure.

Combining fuzzy domain ontology with fuzzy linguistic variable ontology, we obtain the threelayered ontology structure shown in figure 1, which could represent fuzzy knowledge more effectively.

\section{Application to transportation knowledge modeling}


Intelligent Transportation System (ITS), a developing conformation of transportation system in the information times, bas been integrating a variety of advanced technologies, especially information technology [11]. The core of ITS is intelligent, so it is necessary to utilize and share the knowledge efficiently. At present, the transportation decision system based on knowledge can explain, forecast and testify the transportation situation and provides decision support for transportation supervisor and participants with a series of reasoning and calculation, and then the value of transportation information can be increased and the service of transportation system can be extended [12].

ITS lays stress on the relationship between human, vehicle and road. There a lot of fuzzy phenomenon from human factor. For instance, in traffic information service domain, people pay attention to message such as weather information, road information, accident information and gas station information etc. It is sufficient for travelers to obtain some message in linguistic values rather than in accurate numeric values, such as weather information, road information etc. Linguistic values for weather include "overcast", "cloudy", "fine”, "rainy", "snow" etc, and linguistic values for road surface condition include "dryness", “dampness", "seeper", "firn” etc. These linguistic values have uncertainty and are fuzzy concepts.

At the same time, traffic accident management domain and vehicle safety and driving assistance domain need also these messages. To share and reuse knowledge between traffic application systems, it is necessary to construct knowledge ontology from traffic information.

Using the fuzzy ontology model presented above, we construct knowledge ontology for urban road accident analysis and forecasting. The ontology structure is shown in figure 2, including set of concepts, set of properties and set of fuzzy linguistic variable ontologies, in which some fuzzy linguistic variable ontologies can be shared by other traffic application systems. The relation between concept and property is "property of", and the relation between property and fuzzy linguistic variable ontology is "value of". At the same time, every set has own relationships respectively. Figure 3 shows the concepts hierarchy structure derived from the relation of "kind of”.

\section{Conclusions}

Handling uncertainty of information and knowledge, fuzzy ontology expands the application domain of ontology. It is useful to share and reuse fuzzy knowledge among fuzzy systems on the semantic web.
In this paper we have proposed the fuzzy domain ontology model and the fuzzy linguistic variables ontology model to represent fuzzy knowledge in fuzzy systems. The fuzzy linguistic variables ontology models focus on essential semantic relationships between fuzzy concepts, including set relation, order relation and equivalence relation, which facilitates the ontology mapping and integrating.

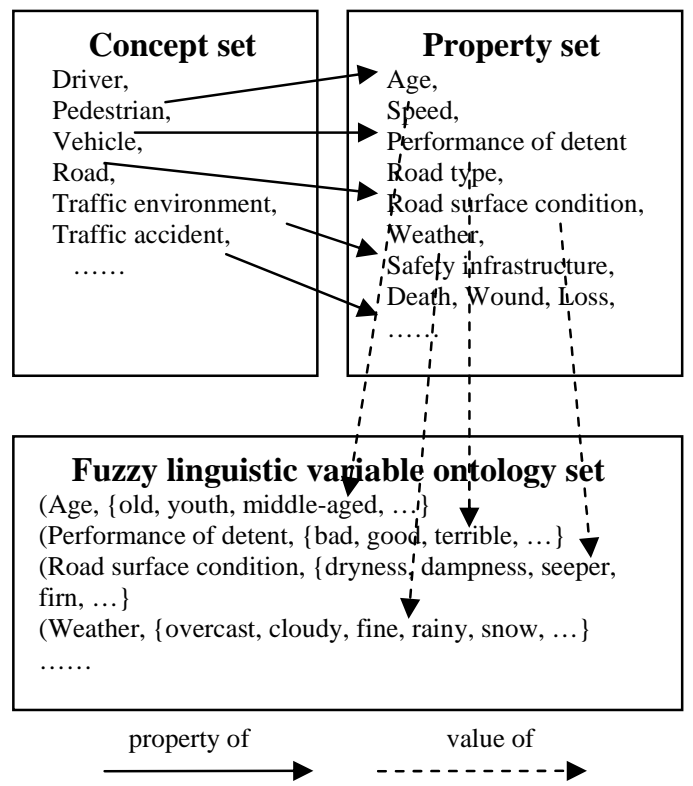

Fig. 2: Ontology structure for transportation knowledge.

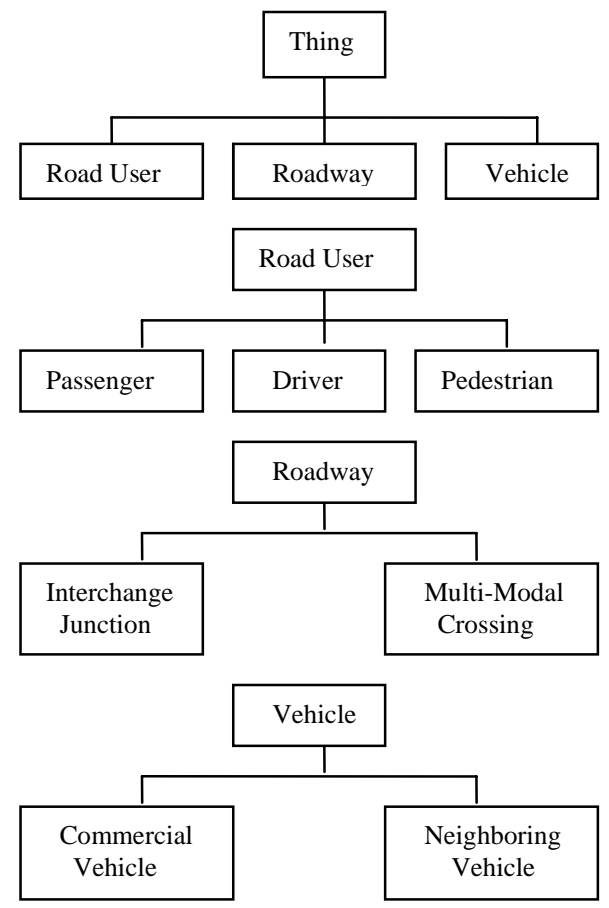

Fig.3: Concepts hierarchy structure 
Our further researches lay on the automatic generation of fuzzy ontology from fuzzy systems and the integration among standard ontology and fuzzy ontology.

\section{References}

[1] D. Fensel, F. van Harmelen, I. Horrocks, D.L.McGuinness and P.F. Patel-Schneider, OIL: An ontology infrastructure for the semantic web. IEEE Intelligent Systems, 16 (2):38-45, 2001.

[2 ] T. Berners-Lee, Semantic web road map, W3C Design Issues, 1998 http://www.w3.org/DesignIssues/Semantic.html.

[3 ] L.A.Zadeh, J.Kacprzyk, Fuzzy logic for the management of uncertainty, John Wiley \& Sons, Inc, 1992.

[4 ] D.H.Widyantoro and J.Yen, A fuzzy ontologybased abstract search engine and its user studies. In: Proceedings of the 10th IEEE International Conference on Fuzzy Systems, Melbourne, Australia, pp. 1291- 1294, 2001.

[5 ] D.Parry, A fuzzy ontology for medical document retrieval. In: Proceedings of the Second Workshop on Australasian Information Security, Data Mining and Web Intelligence, and Software Internationalization, Dunedin, New Zealand, pp. 121- 126, 2004.

[6] C.S. Lee, Z.W. Jian and L.K. Huang, A fuzzy ontology and its application to news summarization. IEEE Transactions on Systems, Man and Cybernetics (Part B), 35(5): 859- 880, 2005.

[7] C. S. Lee, Y. J. Chen and Z. W. Jian, Ontology based fuzzy event extraction agent for chinese enews summarization. Expert Systems With Applications, 25(3):431-447, 2003.

[8] Q. T. Tho, S. C. Hui, A. C. M. Fong and T. H. Cao, Automatic fuzzy ontology generation for semantic web. IEEE Transactions on Knowledge and Data Engineering, 18(6):842- 856, 2006.

[9] Muhammad Abulaish, Lipika Dey, Interoperability among distributed overlapping ontologies - a fuzzy ontology framework. In: Proceedings of the 2006 IEEE/WIC/ACM International Conference on Web Intelligence (WI 2006 Main Conference Proceedings)(WI'06), 2006.

[10] D.Z. Kang, B.W. Xu, J.J. Lu and Y.H. Li, Description logics for fuzzy ontologies on semantic web. Journal of Southeast University (English Edition), 22(3):343 - 347, 2006.

[11] Lino Figueiredo, Isabel Jesus, J. A. Tenreiro Machado, Jose Rui Ferreira, J. L. Martins de Carvalho, Towards the development of intelligent transportation systems. In: Proceedings of IEEE
Intelligent Transportation Systems Conference, USA, pp.1206-1211, 2001.

[12] Y. Li, J. Zhai and Y. Chen, Using ontology to achieve the semantic integration of the intelligent transport system. In: Proceedings of 2005 International Conference on Management Science \& Engineering (12th) (Volume III), pp. 2528-2532, 2005. 